TRANSACTIONS OF THE

AMERICAN MATHEMATICAL SOCIETY

Volume 358, Number 8 , Pages 3385-3398

S 0002-9947(06)04119-5

Article electronically published on February 14, 2006

\title{
CURVILINEAR BASE POINTS, LOCAL COMPLETE INTERSECTION AND KOSZUL SYZYGIES IN BIPROJECTIVE SPACES
}

\author{
J. WILLIAM HOFFMAN AND HAO HAO WANG
}

\begin{abstract}
Let $I=\left\langle f_{1}, f_{2}, f_{3}\right\rangle$ be a bigraded ideal in the bigraded polynomial $\operatorname{ring} k[s, u ; t, v]$. Assume that $I$ has codimension 2. Then $Z=\mathbb{V}(I) \subset \mathbf{P}^{1} \times \mathbf{P}^{1}$ is a finite set of points. We prove that if $Z$ is a local complete intersection, then any syzygy of the $f_{i}$ vanishing at $Z$, and in a certain degree range, is in the module of Koszul syzygies. This is an analog of a recent result of Cox and Schenck (2003).
\end{abstract}

\section{INTRODUCTION}

Let $X$ be a smooth algebraic surface over a field $k$, and let $\mathcal{I} \subset \mathcal{O}_{X}$ be a coherent sheaf of ideals. Suppose that $Z=\mathbb{V}(\mathcal{I})$ is a finite set, an assumption that holds throughout this paper. Often, we call the points of $Z$ base points for $\mathcal{I}$. The reason for the terminology is that in our examples $\mathcal{I}$ will arise from an ideal $I$ in a polynomial ring generated by polynomials defining a rational map $X \rightarrow \mathbf{P}^{N}$. Then $Z$ will be the base point locus of this map.

Definition 1.1. We say a coherent ideal sheaf $\mathcal{I} \subset \mathcal{O}_{X}$ is curvilinear at $p \in Z$, if in the completion $\widehat{\mathcal{O}}_{X, p}$, there are analytical coordinates $u, v$ so $\widehat{\mathcal{O}}_{X, p}=k[[u, v]]$ such that $\widehat{\mathcal{I}}_{p}=\left\langle u, v^{k}\right\rangle$, for some $k \geq 1$.

Remark 1.2. In case $X=\mathbf{P}^{2}, \mathcal{I}$ will come from a homogeneous ideal $I \subset k[x, y, z]$, and we will say $I$ is curvilinear at $p$ if the sheaf it generates is curvilinear. Similarly, if $X=\mathbf{P}^{1} \times \mathbf{P}^{1}, \mathcal{I}$ will come from a bihomogeneous ideal $I \subset k[s, u, t, v]$. We will also use the terminology that the base point $p \in Z=\mathbb{V}(\mathcal{I})$ is curvilinear.

Definition 1.3. The base points are local complete intersection (LCI) if for every point $p \in Z, I \mathcal{O}_{X, p}$ is a complete intersection ideal, i.e., generated by a regular sequence in $\mathcal{O}_{X, p}$.

Remark 1.4. If $I$ is curvilinear at $p$, then $I$ is LCI at $p$.

Recall that given elements $r_{1}, \cdots, r_{n}$ in any commutative ring $R$, a syzygy $\left(a_{1}, \cdots, a_{n}\right)$ is a relation $a_{1} r_{1}+\cdots+a_{n} r_{n}=0$ for $a_{i} \in R$. A Koszul syzygy is one of the form $\left(r_{j}\right) r_{i}+\left(-r_{i}\right) r_{j}=0$ for $i \neq j$. Let $\operatorname{Syz}\left(r_{1}, \cdots, r_{n}\right)$ be the submodule of $R^{n}$ generated by the syzygies. Let $\operatorname{Kos}\left(r_{1}, \cdots, r_{n}\right) \subset \operatorname{Syz}\left(r_{1}, \cdots, r_{n}\right)$ be the

Received by the editors May 19, 2003 and, in revised form, May 7, 2004.

2000 Mathematics Subject Classification. Primary 14Q10; Secondary 13D02, 14 Q05.

Key words and phrases. Base points, local complete intersection, syzygy, saturation, projective space.

The authors thank William Adkins and David Cox for numerous discussions and suggestions. 
submodule generated by the Koszul syzygies. We refer to an arbitrary element of $\operatorname{Kos}\left(r_{1}, \cdots, r_{n}\right)$ as a Koszul syzygy.

For the rest of this introduction, we will discuss the special case $R=k[x, y, z]$, and $I$ is generated by homogeneous forms with $Z=\mathbb{V}(I) \subset \mathbf{P}^{2}$ a finite set of points. These syzygy modules are graded and there is no loss in generality in considering syzygies that are homogeneous.

Definition 1.5. A syzygy $\left(a_{1}, \cdots, a_{n}\right) \in \operatorname{Syz}\left(r_{1}, \cdots, r_{n}\right)$ vanishes at all base points of $I$ if, for each $i, a_{i} \in I^{\text {sat }}$, where $I^{\text {sat }}=\left\{r \in R:\langle x, y, z\rangle^{k} r \subset I\right.$ for some $\left.k\right\}$. If $a_{i}$ is homogeneous of degree $d_{i}$, this is equivalent to saying that $a_{i}$ belongs to $I \mathcal{O}_{\mathbf{P}^{2}, p}\left(d_{i}\right)$ for all $p \in Z$, for all $i$, where $\mathcal{O}_{\mathbf{P}^{2}}(d)$ is the standard invertible sheaf, $d \in \mathbb{Z}$. This is also equivalent to saying that each $a_{i}$ is a global section of $I \mathcal{O}_{\mathbf{P}^{2}}\left(d_{i}\right)=\mathcal{I}\left(d_{i}\right)$.

The equivalence of the two conditions in the above definition follows from the facts

$$
I_{d_{i}}^{\text {sat }}=H^{0}\left(\mathbf{P}^{2}, I \mathcal{O}_{\mathbf{P}^{2}}\left(d_{i}\right)\right) \text { and } I \mathcal{O}_{\mathbf{P}^{2}, p}\left(d_{i}\right)=\mathcal{O}_{\mathbf{P}^{2}, p}\left(d_{i}\right) \text { for all } p \notin Z \text {. }
$$

Now consider an ideal $I=\langle a, b, c\rangle \subset R$, where $a, b, c$ are homogeneous polynomials of degree $m$. Cox has proved following theorem:

Theorem 1.6 ([1, Theorem 5.5]). Let $k$ be algebraically closed. If all the base points of $I$ are curvilinear, then every syzygy on $a, b, c$ which vanishes on $Z$ is a Koszul syzygy.

Cox proves this under the assumption that $k=\mathbb{C}$, but the arguments are more general. We will prove a version of this theorem for the bigraded case in Section 2 , Cox and Schenck [3] have proved the following result which strengthens Theorem 1.6 .

Theorem 1.7 ([3, Theorem 1.7]). If $I=\left\langle f_{1}, f_{2}, f_{3}\right\rangle \subset R=k[x, y, z]$, where $f_{i}$ is a homogeneous polynomial, and if $I$ has codimension 2 , then the module of syzygies of $f_{1}, f_{2}, f_{3}$ vanishing at $Z=\mathbb{V}(I)$ is generated by the Koszul syzygies if and only if $I$ is a local complete intersection.

We will extend this result to the bigraded case in Section 3 The proofs of Theorems 1.6 and 1.7 are quite different; the former is geometric, the latter algebraic. We present arguments that parallel the proofs of the above theorems. Even though the first follows logically from the second, we present both arguments, because they are so different. Also, there are some significant differences from the case considered by Cox and Schenck. In fact we can only prove that those syzygies vanishing at the base points, and of sufficiently high degree, are Koszul syzygies. We present an example to show that, in the bigraded case, not every syzygy vanishing at the base points is Koszul. The key difference between their situation and ours is that, for any invertible sheaf $\mathcal{L}$ on $\mathbf{P}^{2}$, we have $H^{1}\left(\mathbf{P}^{2}, \mathcal{L}\right)=0$, whereas this vanishing does not hold on $\mathbf{P}^{1} \times \mathbf{P}^{1}$. The results in this paper are used in an essential way in Wang's thesis, [13].

\section{Curvilinear base points and Koszul syzygies}

In this section, $k$ is an algebraically closed field. Let $R=k[s, u, t, v]$ be the bigraded coordinate ring of $\mathbf{P}^{1} \times \mathbf{P}^{1}$, where $s, u$ have bidegree $(1,0)$ and $t, v$ have bidegree $(0,1)$. Let $\mathbf{m}=\langle s t, s v, u t, u v\rangle$, the irrelevant ideal in $R$. Consider an ideal 
$I=\langle a, b, c\rangle \subset R$, where $a, b, c$ are homogeneous of the same degree $(m, n)$. Let $Z=\mathbb{V}(a, b, c) \subset \mathbf{P}^{1} \times \mathbf{P}^{1}$, which is assumed to be a finite set. Let $r_{1}, \cdots, r_{n} \in R$ be bihomogeneous elements, and consider syzygies as before. All our syzygy modules will be bigraded, and syzygies themselves will be taken to be bihomogeneous.

Definition 2.1. A syzygy $\left(a_{1}, \cdots, a_{n}\right) \in \operatorname{Syz}\left(r_{1}, \cdots, r_{n}\right)$ vanishes at all base points of $I$ if, for each $i, a_{i} \in I^{\mathbf{m s a t}}=\left\{r \in R: \mathbf{m}^{k} r \subset I\right.$, for some $\left.k\right\}$. If $a_{i}$ is homogeneous of bidegree $\left(d_{i}, d_{i}^{\prime}\right)$, this is equivalent to saying that $a_{i} \in I \mathcal{O}_{\mathbf{P}^{1} \times \mathbf{P}^{1}, p}\left(d_{i}, d_{i}^{\prime}\right)$, for all $p \in Z$, where $\mathcal{O}_{\mathbf{P}^{1} \times \mathbf{P}^{1}}\left(d, d^{\prime}\right)$ is the standard invertible sheaf, $d, d^{\prime} \in \mathbb{Z}$. This is also equivalent to saying that each $a_{i}$ is a global section of $I \mathcal{O}_{\mathbf{P}^{1} \times \mathbf{P}^{1}}\left(d_{i}, d_{i}^{\prime}\right)=\mathcal{I}\left(d_{i}, d_{i}^{\prime}\right)$.

The equivalence follows as before, noting that

$$
I_{d_{i}, d_{i}^{\prime}}^{\mathrm{msaa}}=H^{0}\left(\mathbf{P}^{1} \times \mathbf{P}^{1}, I \mathcal{O}_{\mathbf{P}^{1} \times \mathbf{P}^{1}}\left(d_{i}, d_{i}^{\prime}\right)\right)
$$

and

$$
I \mathcal{O}_{\mathbf{P}^{1} \times \mathbf{P}^{1}, p}\left(d_{i}, d_{i}^{\prime}\right)=\mathcal{O}_{\mathbf{P}^{1} \times \mathbf{P}^{1}, p}\left(d_{i}, d_{i}^{\prime}\right) \quad \text { for all } p \notin Z .
$$

We shall be concerned with the syzygies $(A, B, C)$ on the generators of $I=(a, b, c)$.

Remark 2.2. Consider the exact sequence

$$
0 \longrightarrow \operatorname{Syz}(a, b, c) \longrightarrow \bigoplus_{i=1}^{3} R\left(-d_{i},-d_{i}^{\prime}\right) \stackrel{(a, b, c)}{\longrightarrow} I \longrightarrow 0 .
$$

$\operatorname{Syz}(a, b, c)$ is a bigraded submodule of $\bigoplus_{i=1}^{3} R\left(-d_{i},-d_{i}^{\prime}\right)$, and we say $(A, B, C) \in$ $\operatorname{Syz}(a, b, c)_{k, l}$ has bidegree $(k, l)$. Note that, as polynomials, the bidegree of $A$ is $\left(k-d_{1}, l-d_{1}^{\prime}\right)$, since $A \in R\left(-d_{1},-d_{1}^{\prime}\right)_{k, l}$, etc. In other words, the polynomial expression $A a+B b+C c$ has bidegree $(k, l)$. If all $\left(d_{i}, d_{i}^{\prime}\right)$ are equal to a fixed pair $(m, n)$, then a bihomogeneous syzygy $(A, B, C)$ of bidegree $(k, l)$ will have $A, B, C$ all bihomogeneous of the same bidegree $(k-m, l-n)$. Some authors call this a syzygy of bidegree $(k-m, l-n)$. We will call $(A, B, C)$ a syzygy of pure degree $(k-m, l-n)$.

Proposition 2.3. Let $a, b, c \in R$ be bihomogeneous polynomials of bidegree $(m, n)$ and suppose $\mathbb{V}(a, b, c) \subset \mathbf{P}^{1} \times \mathbf{P}^{1}$ is finite. Suppose that all the base points of $a, b, c$ are curvilinear. Then every syzygy $A a+B b+C c=0$ with pure bidegree $(k, l)$, where $(k-2 m+1)(l-2 n+1) \geq 0$, and vanishing at the base points is a Koszul syzygy. That is, there exists $h_{1}, h_{2}, h_{3}$ of bidegree $(k-m, l-n)$ such that

$$
\begin{aligned}
& A=h_{1} c+h_{2} b, \\
& B=-h_{2} a+h_{3} c, \\
& C=-h_{1} a-h_{3} b .
\end{aligned}
$$

Note. When $\mathbb{V}(a, b, c)=\emptyset$, this is proved in [2]. The analogous result for $\phi: \mathbf{P}^{2} \longrightarrow$ $\mathbf{P}^{3}$, namely Theorem[1.6, is proved in [1, but with no restriction at all on the degree of the syzygies. Note also that the Koszul syzygies themselves have pure degree $(m, n)$ and satisfy the conditions of the proposition.

Proof. In [1, using the theory of toric varieties, Cox constructed a projective birational map (blowing up) $\pi: X \rightarrow Q=\mathbf{P}^{1} \times \mathbf{P}^{1}$ with the following properties:

1. $X$ is a smooth projective surface.

2. $I_{Z}^{\prime}=I \mathcal{O}_{X}=\mathcal{O}_{X}(-E)$ for a divisor $E$ on $X$.

3. The canonical class $K_{X}=\pi^{*} K_{Q}+E$, where $K_{Q}=\mathcal{O}_{Q}(-2,-2)$ is the canonical class of $Q$. 
We have $a, b, c \in \Gamma\left(Q, \mathcal{I}_{Z} \otimes \mathcal{O}_{Q}(m, n)\right) \subset R_{m, n}$, and we get their proper transform

$$
\tilde{a}, \tilde{b}, \tilde{c} \in \Gamma\left(X, \mathcal{I}_{Z}^{\prime} \otimes \pi^{*} \mathcal{O}_{Q}(m, n)\right) .
$$

Let $L_{1}, L_{2}$ be generators of $\operatorname{Pic}(Q)$, i.e., generating lines on $Q$ which are chosen to miss $Z$. By abuse of notation, we let $L_{1}, L_{2} \subset X$ be the inverse images on $X$. Hence

$$
\tilde{a}, \tilde{b}, \tilde{c} \in \Gamma\left(X, \mathcal{O}_{X}\left(m L_{1}+n L_{2}-E\right)\right) .
$$

These are without base points, i.e.,

$$
\mathcal{F}:=\mathcal{O}_{X}\left(E-m L_{1}-n L_{2}\right)^{3} \stackrel{(\tilde{a}, \tilde{b}, \tilde{c})}{\longrightarrow} \mathcal{O}_{X} \longrightarrow 0
$$

is onto. Build the Koszul complex on this, i.e.,

$$
0 \rightarrow \bigwedge^{3} \mathcal{F} \rightarrow \bigwedge^{2} \mathcal{F} \rightarrow \mathcal{F} \rightarrow \mathcal{O}_{X} \rightarrow 0
$$

with $\bigwedge^{3} \mathcal{F}=\mathcal{O}_{X}\left(3 E-3 m L_{1}-3 n L_{2}\right)$ and $\bigwedge^{2} \mathcal{F}=\mathcal{O}_{X}\left(2 E-2 m L_{1}-2 n L_{2}\right)^{3}$. This is exact on $X$, because $\tilde{a}, \tilde{b}, \tilde{c}$ have no base points [4, Prop. 17.14(b), p. 439].

Let $A, B, C$ be a syzygy of pure bidegree $(k, l)$ which vanishes at base points, i.e.,

$$
A, B, C \in \Gamma\left(Q, \mathcal{I}_{Z} \otimes \mathcal{O}_{Q}(k, l)\right) .
$$

Their proper transforms are $\tilde{A}, \tilde{B}, \tilde{C} \in \Gamma\left(X, \mathcal{O}_{X}\left(k L_{1}+l L_{2}-E\right)\right)$. Tensor the Koszul sequence (11) with $\mathcal{O}_{X}\left((k+m) L_{1}+(l+m) L_{2}-2 E\right)$ and break the resulting sequence of 4 terms into 2 exact sequences

$$
\begin{aligned}
0 \rightarrow & \mathcal{O}_{X}\left((k-2 m) L_{1}+(l-2 n) L_{2}+E\right) \rightarrow \mathcal{O}_{X}\left((k-m) L_{1}+(l-n) L_{2}\right)^{3} \rightarrow \mathcal{K} \rightarrow 0, \\
& 0 \rightarrow \mathcal{K} \rightarrow \mathcal{O}_{X}\left(k L_{1}+l L_{2}-E\right)^{3} \rightarrow \mathcal{O}_{X}\left((k+m) L_{1}+(l+n) L_{2}-2 E\right) \rightarrow 0 .
\end{aligned}
$$

From the exact cohomology sequence, we see that if

$$
H^{1}\left(X, \mathcal{O}_{X}\left((k-2 m) L_{1}+(l-2 n) L_{2}+E\right)\right)=0,
$$

then the sequence

$$
\begin{gathered}
H^{0}\left(X, \mathcal{O}_{X}\left((k-m) L_{1}+(l-n) L_{2}\right)\right)^{3} \rightarrow H^{0}\left(X, \mathcal{O}_{X}\left(k L_{1}+l L_{2}-E\right)\right)^{3} \\
\stackrel{(\tilde{a}, \tilde{b}, \tilde{c})}{\longrightarrow} H^{0}\left(X, \mathcal{O}_{X}\left((k+m) L_{1}+(l+n) L_{2}-2 E\right)\right)
\end{gathered}
$$

is exact in the middle. But because $\pi_{*} \mathcal{O}_{X}=\mathcal{O}_{Q}$ and $R^{i} \pi_{*} \mathcal{O}_{X}=0$ if $i>0$ (see Lemma 2.4 below), we have that

$$
H^{0}\left(X, \mathcal{O}_{X}\left((k-m) L_{1}+(l-n) L_{2}\right)\right) \cong H^{0}\left(Q, \mathcal{O}_{Q}(k-m, l-n)\right) .
$$

This follows from the Leray spectral sequence. Note that $\mathcal{O}_{X}\left(k L_{1}+l L_{2}\right)=$ $\pi^{*} \mathcal{O}_{Q}(k, l)$. Then $\pi_{*} \mathcal{O}_{X}\left(k L_{1}+l L_{2}\right)=\mathcal{O}_{Q}(k, l) \otimes \pi_{*} \mathcal{O}_{X}$ by the projection formula [6. Ch. III, §8, Ex. 8.3, p. 253].

We can consider the following diagram:

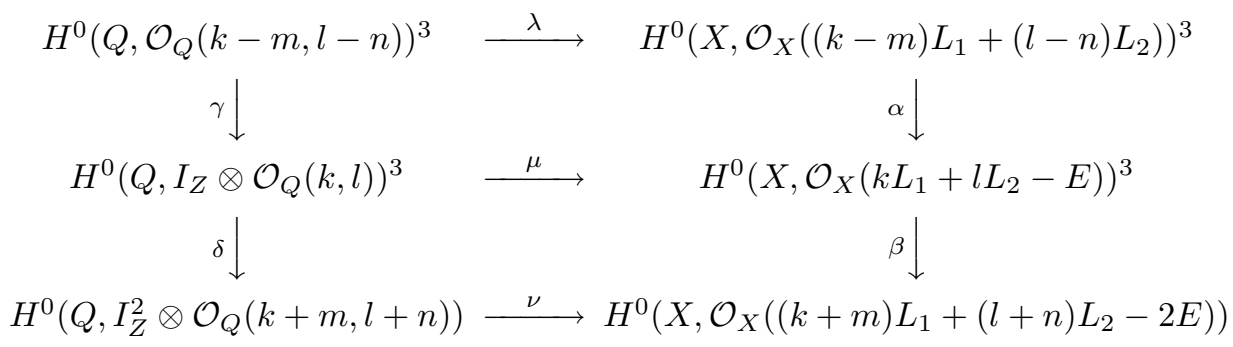


where $\delta=(a, b, c)$ and $\beta=(\tilde{a}, \tilde{b}, \tilde{c})$. We have seen that $\lambda$ is an isomorphism, and clearly, $\mu$ and $\nu$ are injective. Now an element $(A, B, C)$ from the middle term of the first column in the kernel of $\delta$ is precisely a syzygy vanishing at the base points. $A a+B b+C c=0$ implies that $(\tilde{A}, \tilde{B}, \tilde{C})$ is in the image of $\alpha$, since the right sequence is exact in the middle, as we have shown. Thus, $A, B, C$ is in the image of $\gamma$, since the diagram commutes. But this says exactly that $(A, B, C)$ is a Koszul syzygy, as was to be shown.

It remains to check that $H^{1}\left(X, \mathcal{O}_{X}\left((k-2 m) L_{1}+(l-2 n) L_{2}+E\right)\right)=0$. By Serre duality, this is dual to

$$
H^{1}\left(X, \mathcal{O}_{X}\left(-(k-2 m) L_{1}-(l-2 n) L_{2}-E\right) \otimes \mathcal{K}_{X}\right),
$$

but $\mathcal{K}_{X} \cong \pi^{*} \mathcal{K}_{Q}+E$ and $\mathcal{K}_{Q} \cong \mathcal{O}_{Q}(-2,-2)$. Hence, the above is

$H^{1}\left(X, \mathcal{O}_{X}\left((2 m-k-2) L_{1}+(2 n-l-2) L_{2}\right)\right)=H^{1}\left(X, \pi^{*} \mathcal{O}_{Q}(2 m-k-2,2 n-l-2)\right)$.

From the Leray spectral sequence, Lemma 2.4 below, and the projection formula, we get

$$
H^{1}\left(X, \pi^{*} \mathcal{O}_{Q}(2 m-k-2,2 n-l-2)\right) \cong H^{1}\left(Q, \mathcal{O}_{Q}(2 m-k-2,2 n-l-2)\right) .
$$

Now applying the Künneth formula, [10],

$$
\begin{aligned}
& H^{1}\left(\mathbf{P}^{1} \times \mathbf{P}^{1}, \mathcal{O}_{\mathbf{P}^{1} \times \mathbf{P}^{1}}(2 m-k-2,2 n-l-2)\right) \\
& =\bigoplus_{i+j=1} H^{i}\left(\mathbf{P}^{1}, \mathcal{O}(2 m-k-2)\right) \otimes H^{j}\left(\mathbf{P}^{1}, \mathcal{O}(2 n-l-2)\right) .
\end{aligned}
$$

By Serre's computation of the cohomology of projective space and this last one clearly is 0 , when $k=2 m-1, \forall l$, or $l=2 n-1, \forall k$, or $k>2 m-1, l>2 n-1$, or $k<2 m-1, l<2 n-1$, i.e., $(k-2 m+1)(l-2 n+1) \geq 0$.

\section{Lemma 2.4 .}

$$
\pi_{*} \mathcal{O}_{X}=\mathcal{O}_{Q}, \quad R^{i} \pi_{*} \mathcal{O}_{X}=0 \text { if } i>0 .
$$

Proof. Since $\pi$ is a proper birational morphism of smooth surfaces, Zariski's Factorization Theorem [6. Corollary 5.4, p. 411] states that we may factor $\pi$ into a succession of maps $\pi_{i}: X_{i} \rightarrow X_{i-1}$, where $X_{0}=Q, X_{n}=X$, and each $\pi_{i}$ is a blowing up of one point (monoidal transformation). The result now follows by induction on $n$, using the spectral sequence of a composite functor and the fact, shown by direct computation, that it is true for a monoidal transformation. In fact, this result is a well-known fact for any succession of blowing-ups with smooth centers.

Remark 2.5. The reader may wonder where the hypothesis of curvilinear base point has come into the above argument. It occurs in the existence of the blowing-up $\pi$ with the properties enumerated at the beginning of the proof. Starting with any ideal $I$ one may construct a blowing-up to a smooth $X$ with the property that $I \mathcal{O}_{X}=\mathcal{O}_{X}(-E)$ for a divisor $E$ on $X$. This is a general fact for varieties in any dimension, at least in characteristic 0, by Hironaka's theorems, and one may even assume $E$ to have normal crossings. In our case, for surfaces, Zariski's theorem utilized above makes it clear that $K_{X}=\pi^{*} K_{Q}+E^{\prime}$ for some divisor $E^{\prime}$ supported in the same set as $E$, but the key fact in the curvilinear case is that $E=E^{\prime}$, an exact equality including multiplicities. In view of the fact that the conclusion of the theorem holds for $I$ a local complete intersection, it might be of interest to extend 
the above proof to that case. This requires some analysis of the divisor $E^{\prime}$ and its relation to $E$ in that situation.

Example 2.6. The following example shows that not all syzygies vanishing at the base points are Koszul syzygies: Let $I=\left\langle u^{2} t v, u^{2} t^{2}+s u v^{2}, s^{2} t v\right\rangle$. The base points of ideal $I$ are

$$
p=(0: 1 ; 0: 1), p^{\prime}=(1: 0 ; 1: 0)
$$

and

$$
I_{p}=\langle s, t\rangle, I_{p^{\prime}}=\left\langle v, u^{2}\right\rangle .
$$

These are curvilinear. The (pure) degrees of the generators are $(2,2)$, so our theorem claims that all syzygies vanishing at the base points and either of degrees $\geq(3,3)$ or $\leq(3,3)$ are generated by Koszul syzygies. Using Singular [5], we found generators for the syzygy module vanishing at the base points as the rows of the following matrix:

$$
\left[\begin{array}{ccc}
u^{2} t^{2}+s u v^{2} & -u^{2} t v & 0 \\
s^{2} t v & 0 & -u^{2} t v \\
0 & s^{2} t v & -u^{2} t^{2}-s u v^{2} \\
u t^{3} v+s t v^{3} & -u t^{2} v^{2} & 0 \\
s t^{3} v & -s t^{2} v^{2} & u t v^{3} \\
s^{2} t^{2} v & -s^{2} t v^{2} & s u v^{3} \\
s^{3} u v & 0 & -s u^{3} v \\
s^{2} u^{2} t & 0 & -u^{4} t \\
s^{3} u^{2} & 0 & -s u^{4}
\end{array}\right]
$$

The Koszul syzygies are generated by the first three rows. The sixth row is of degree $(2,3)$, and it is generated by the Koszul syzygies since

$$
\left(s^{2} t^{2} v,-s^{2} t v^{2}, s u v^{3}\right)=t\left(s^{2} t v, 0,-u^{2} t v\right)-v\left(0, s^{2} t v,-u^{2} t^{2}-s u v^{2}\right) .
$$

The syzygies

$$
\begin{gathered}
\left(u t^{3} v+s t v^{3},-u t^{2} v^{2}, 0\right),\left(s t^{3} v,-s t^{2} v^{2}, u t v^{3}\right),\left(s^{3} u v, 0,-s u^{3} v\right) \\
\left(s^{2} u^{2} t, 0,-u^{4} t\right),\left(s^{3} u^{2}, 0,-s u^{4}\right)
\end{gathered}
$$

are of degree $(1,4),(1,4),(4,1),(4,1),(5,0)$. They are not Koszul, because their degrees forbid this. But we can look at multiples of say $\mathbf{v}=\left(s t^{3} v,-s t^{2} v^{2}, u t v^{3}\right)$, which is of degree $(1,4)$. Since

$$
s\left(s t^{3} v,-s t^{2} v^{2}, u t v^{3}\right)=t^{2}\left(s^{2} t v, 0,-u^{2} t v\right)-t v\left(0, s^{2} t v,-u^{2} t^{2}-s u v^{2}\right),
$$

we see that $s^{2} \mathbf{v}$ and $s u \mathbf{v}$, of degrees $(3,4)$, are in the module of Koszul syzygies, as is claimed by our theorem. The syzygy $u \mathbf{v}$, of degree $(2,4)$, is not generated by Koszul syzygies, but

$$
u^{2}\left(s t^{3} v,-s t^{2} v^{2}, u t v^{3}\right)=s t v\left(u^{2} t^{2}+s u v^{2},-u^{2} t v, 0\right)-u v^{2}\left(s^{2} t v, 0,-u^{2} t v\right),
$$

so $u^{2} \mathbf{v}$, of bidegree $(3,4)$, is in the module of Koszul syzygies. 


\section{Local COMPLETE INTERSECTION AND Koszul SyZyGies}

Now assume that $k$ is any field and consider an ideal $I=\left\langle f_{1}, f_{2}, f_{3}\right\rangle \subset R=$ $k[s, u, t, v]$, where $f_{i}$ is bihomogeneous of degree $\left(d_{i}, d_{i}^{\prime}\right)$. The $f_{i}$ form a regular sequence in $R$ if and only if the following Koszul complex is exact:

$$
\begin{aligned}
0 \rightarrow & R\left(-\sum_{i=1}^{3} d_{i},-\sum_{i=1}^{3} d_{i}^{\prime}\right) \stackrel{\left[\begin{array}{c}
f_{3} \\
-f_{2} \\
f_{1}
\end{array}\right]}{\longrightarrow} \bigoplus_{i<j} R\left(-d_{i}-d_{j},-d_{i}^{\prime}-d_{j}^{\prime}\right) \\
& \stackrel{\left[\begin{array}{ccc}
f_{2} & f_{3} & 0 \\
-f_{1} & 0 & f_{3} \\
0 & -f_{1} & -f_{2}
\end{array}\right]}{\longrightarrow} \bigoplus_{i=1}^{3} R\left(-d_{i},-d_{i}^{\prime}\right) \stackrel{f_{1} f_{2} f_{3}}{\longrightarrow} I \rightarrow 0 .
\end{aligned}
$$

We will discuss the situation when $Z=\mathbb{V}(I) \subset \mathbf{P}^{1} \times \mathbf{P}^{1}$ is a zero-dimensional subscheme, thus $I=\left\langle f_{1}, f_{2}, f_{3}\right\rangle$ has codimension two in $R$. We call $Z$ the base point locus of $f_{1}, f_{2}, f_{3}$. If $I$ has codimension two, $f_{1}, f_{2}, f_{3}$ will no longer be a regular sequence: since $R$ is Cohen-Macaulay, the depth of $I$ equals the codimension of $I$, so that a maximal $R$-sequence in $I$ has length two.

Lemma 3.1. If $I=\left\langle f_{1}, f_{2}, f_{3}\right\rangle$ has codimension two in $R$, then the sequence (2) is exact except at $\bigoplus_{i=1}^{3} R\left(-d_{i},-d_{i}^{\prime}\right)$. In particular, the Koszul complex of $f_{1}, f_{2}, f_{3}$ gives the exact sequences

$$
0 \rightarrow R\left(-\sum_{i=1}^{3} d_{i},-\sum_{i=1}^{3} d_{i}^{\prime}\right) \rightarrow \bigoplus_{i<j} R\left(-d_{i}-d_{j},-d_{i}^{\prime}-d_{j}^{\prime}\right) \rightarrow \bigoplus_{i=1}^{3} R\left(-d_{i},-d_{i}^{\prime}\right)
$$

and

$$
\bigoplus_{i=1}^{3} R\left(-d_{i},-d_{i}^{\prime}\right) \rightarrow I \rightarrow 0 .
$$

Proof. The exactness of the first sequence follows from $\operatorname{depth}(I)=\operatorname{codim}(I)=2$ and the Buchsbaum-Eisenbud exactness theorem (4, p. 500]).

Definition 3.2. A Koszul syzygy on $f_{1}, f_{2}, f_{3}$ is an element of the submodule

$$
K \subset \bigoplus_{i=1}^{3} R\left(-d_{i},-d_{i}^{\prime}\right)
$$

generated by the columns of the matrix

$$
\left[\begin{array}{ccc}
f_{2} & f_{3} & 0 \\
-f_{1} & 0 & f_{3} \\
0 & -f_{1} & -f_{2}
\end{array}\right]
$$

Corollary 3.3. If $I=\left\langle f_{1}, f_{2}, f_{3}\right\rangle$ is a codimension two ideal, then we have an exact sequence

$$
0 \rightarrow R\left(-\sum_{i=1}^{3} d_{i},-\sum_{i=1}^{3} d_{i}^{\prime}\right) \rightarrow \bigoplus_{i<j} R\left(-d_{i}-d_{j},-d_{i}^{\prime}-d_{j}^{\prime}\right) \rightarrow K \rightarrow 0 .
$$

According to [8, Theorem 4.10], $K$ is strongly $\left(p, p^{\prime}\right)$-regular, where

$$
p=\max \left(d_{i}+d_{j}\right), \quad p^{\prime}=\max \left(d_{i}^{\prime}+d_{j}^{\prime}\right)
$$

for $1 \leq i<j \leq 3$. 
Note. $K$ is a submodule of the syzygy module $S$ defined by the exact sequence

$$
0 \rightarrow S \rightarrow \bigoplus_{i=1}^{3} R\left(-d_{i},-d_{i}^{\prime}\right) \rightarrow I \rightarrow 0
$$

In fact, $K$ is a proper submodule of $S$, because if $K=S$, we would have exactness of the sequence (2), which is equivalent to the $f_{i}$ forming a regular sequence, which as we have remarked, is impossible.

Analogous to [12, p. 32], we have the following definition.

Definition 3.4. A bigraded submodule $M$ of a finitely generated free $R$-module $F$ is saturated if

$$
M=\{x \in F \mid \mathbf{m} x \subset M\} .
$$

We define the saturation of $M$ to be

$$
M^{\text {msat }}=\left\{x \in F \mid \mathbf{m}^{k} x \subset M, \text { for some } k\right\},
$$

where $\mathbf{m}=\langle s t, s v, u t, u v\rangle$. We will use the notation $M^{\text {sat }}$ for this, the ideal $\mathbf{m}$ being understood.

Remark 3.5. One can easily show that $M$ is saturated if and only if $M=M^{\text {sat }}$.

Proposition 3.6. Let $M$ be a bigraded submodule of a free $R=k[s, u, t, v]$-module of finite rank $F$. Let $\mathcal{M}$ be the corresponding coherent sheaf on $X=\mathbf{P}^{1} \times \mathbf{P}^{1}$. Then

$$
M_{k, l}^{s a t}=H^{0}(X, \mathcal{M}(k, l)) .
$$

Proof. For any finitely generated bigraded $R$-module we have an exact sequence (see [9])

$$
0 \longrightarrow H_{\mathbf{m}}^{0}(M) \longrightarrow M \longrightarrow \bigoplus_{(a, b) \in \mathbb{Z}^{2}} H^{0}(X, \mathcal{M}(a, b)) \longrightarrow H_{\mathbf{m}}^{1}(M) \longrightarrow 0 .
$$

We will show that $H_{\mathbf{m}}^{i}(M)=0$ when $i=0,1$ if $M$ is saturated. This is sufficient since both $M$ and $M^{\text {sat }}$ generate the same sheaf. Since $H_{\mathbf{m}}^{i}(R)=0$, for $i=0,1$, and $M$ is a submodule of a free $R$-module, it is clear that we have a vanishing for $i=0$, which does not depend on $M$ being saturated. The long exact cohomology sequence for

$$
0 \longrightarrow M \longrightarrow F \longrightarrow F / M \longrightarrow 0
$$

has a piece

$$
H_{\mathbf{m}}^{0}(F) \longrightarrow H_{\mathbf{m}}^{0}(F / M) \longrightarrow H_{\mathbf{m}}^{1}(M) \longrightarrow H_{\mathbf{m}}^{1}(F) .
$$

Since the extreme terms are zero, we get an isomorphism

$$
H_{\mathbf{m}}^{1}(M) \cong H_{\mathbf{m}}^{0}(F / M) \text {. }
$$

But the right-hand side is clearly $M^{\text {sat }} / M$, proving our claim.

We will use the following well-known results:

Proposition 3.7. Let $R$ be a Noetherian ring, $J \subset R$ an ideal, and $M$ a finitely generated $R$-module. The following are equivalent:

(1) $\operatorname{Ass}(M) \subset \mathbb{V}(J)$.

(2) $\operatorname{Supp}(M) \subset \mathbb{V}(J)$.

(3) There exists $n \geq 0$ such that $J^{n} M=0$.

When this is so, $H_{J}^{0}(M)=M$. 
Proof. The equivalence of (1) and (2) follows from the fact that both $\operatorname{Ass}(M)$ and $\operatorname{Supp}(M)$ have the same minimal elements ([11, Theorem 1, p. 7]). Assume (3) holds, and let $\mathbf{p}$ be a prime ideal in the support of $M$. Let $m / s \in M_{\mathbf{p}}$. If $\mathbf{p} \nsupseteq J$ there would exist $x \in J \backslash \mathbf{p}$, and clearly $x^{n} \in J^{n} \backslash \mathbf{p}$ for any $n \geq 1$. But then $x^{n} m=0$, and this shows that $m / s=0$, showing that $\mathbf{p}$ cannot be in the support of $M$. Conversely, assume (2); then ([11, Proposition 3, p. 5])

$$
\operatorname{Supp}(M)=\mathbb{V}(\operatorname{ann}(M)) \subset \mathbb{V}(J)
$$

shows that $\sqrt{J} \subset \sqrt{\operatorname{ann}(M)}$, from which (3) follows easily.

To see the last statement, note that $H_{J}^{0}(M)=\left\{m \in M: J^{k} m=0\right.$ for some $\left.k\right\}$.

Remark 3.8. If $M$ is a bigraded module over the ring $R=k[s, u, t, v]$, the elements of $\operatorname{Ass}(M)$ are bihomogeneous. Moreover, taking $J=\mathbf{m}$ to be the irrelevant ideal, the conditions of the previous proposition are easily seen to be equivalent to

(4) There exists $m, n$ such that $M_{k, l}=0$ whenever $k \geq m$ and $l \geq n$, which we abbreviate by writing $(k, l) \gg(0,0)$.

Definition 3.9. The bigraded Hilbert polynomial $H(M)$ of a finitely generated bigraded $R$-module $M$ is the unique polynomial in two variables such that

$$
H(M)\left(n, n^{\prime}\right)=\operatorname{dim}_{k} M_{n, n^{\prime}}
$$

for all $n, n^{\prime} \gg 0$, where $M_{n, n^{\prime}}$ is the bigraded piece of $M$ in degree $\left(n, n^{\prime}\right)$.

Note. If $\mathcal{M}$ is the sheaf of modules associated to $M$, for $n, n^{\prime} \gg 0$, we have

$$
H(M)\left(n, n^{\prime}\right)=\operatorname{dim}_{k} M_{n, n^{\prime}}=\operatorname{dim} H^{0}\left(\mathbf{P}^{1} \times \mathbf{P}^{1}, \mathcal{M}\left(n, n^{\prime}\right)\right) .
$$

Remark 3.10. It is obvious that

$$
H(M)=H\left(M^{\text {sat }}\right) .
$$

Lemma 3.11. Let $M \subset N \subset F$ be bigraded submodules where $F$ is free of finite type. If $M$ is saturated, then $M=N$ if and only if $H(M)=H(N)$.

Proof. We will only show that $H(M)=H(N)$ implies that $M=N . H(M)=$ $H(N)$ says that there exist $n, n^{\prime}$ such that $M_{k, k^{\prime}}=N_{k, k^{\prime}}$ for all $k \geq n, k^{\prime} \geq n^{\prime}$. Let $a_{p, p^{\prime}} \in N_{p, p^{\prime}}$, where $p<n$ or $p^{\prime}<n^{\prime}$. We can find an $\alpha$ such that $\mathbf{m}^{\alpha} a_{p, p^{\prime}} \subset$ $N_{k, k^{\prime}}=M_{k, k^{\prime}}$ for some $k \geq n, k^{\prime} \geq n^{\prime}$. Since $M$ is saturated, $M=M^{\text {sat }}$, thus $a_{p, p^{\prime}} \in M_{p, p^{\prime}}$.

We denote the syzygy module by $S$, the Koszul syzygies by $K$ and the module of syzygies for $f_{1}, f_{2}, f_{3}$ vanishing at the base points by $V$.

Lemma 3.12. As submodules of $\bigoplus_{i=1}^{3} R\left(-d_{i},-d_{i}^{\prime}\right), V$ is a saturated submodule, and $K_{k, k^{\prime}}=K_{k, k^{\prime}}^{\mathrm{sat}}$ when $\left(k-\sum_{i=1}^{3} d_{i}+1\right)\left(k^{\prime}-\sum_{i=1}^{3} d_{i}^{\prime}+1\right) \geq 0$.

Proof. We know that $K \subset V \subset \bigoplus_{i=1}^{3} R\left(-d_{i},-d_{i}^{\prime}\right)$. We first consider $V$. By definition, we have

$$
V=S \cap \bigoplus_{i=1}^{3} I^{\mathrm{sat}}\left(-d_{i},-d_{i}^{\prime}\right) .
$$

Note that $S=S^{\text {sat }}$, or equivalently, that $S$ is saturated. To see this, let $(a, b, c) \in$ $R^{3}$ such that $\mathbf{m}(a, b, c) \subset S$. This means that for all $h \in \mathbf{m}$ and $h(a, b, c) \in$ $\operatorname{Syz}\left(f_{1}, f_{2}, f_{3}\right), h\left(a f_{1}+b f_{2}+c f_{3}\right)=0$. But $R$ has no zero divisors, thus $(a, b, c) \in S$, 
which shows that $S$ is saturated. Since the intersection of saturated submodules is saturated, $V$ is saturated.

We will show $K_{k, k^{\prime}}^{\mathrm{sat}}=K_{k, k^{\prime}}$ for all $\left(k-\sum_{i=1}^{3} d_{i}+1\right)\left(k^{\prime}-\sum_{i=1}^{3} d_{i}^{\prime}+1\right) \geq 0$. Let $r=r_{k, k^{\prime}} \in \bigoplus_{i=1}^{3} R\left(-d_{i},-d_{i}^{\prime}\right)$ with bidegree $\left(k-\sum_{i=1}^{3} d_{i}+1\right)\left(k^{\prime}-\sum_{i=1}^{3} d_{i}^{\prime}+1\right) \geq 0$ and which satisfies $\mathbf{m} r \subset K$. We will show that $r \in K$. Let $L=K+R r$. Consider the short exact sequence

$$
0 \rightarrow K \rightarrow L \rightarrow L / K \rightarrow 0 .
$$

We get a long exact sequence in local cohomology:

$$
0 \rightarrow H_{\mathbf{m}}^{0}(K) \rightarrow H_{\mathbf{m}}^{0}(L) \rightarrow H_{\mathbf{m}}^{0}(L / K) \rightarrow H_{\mathbf{m}}^{1}(K) \rightarrow \cdots
$$

Since $K \hookrightarrow \bigoplus_{i=1}^{3} R\left(-d_{i},-d_{i}^{\prime}\right), H_{\mathbf{m}}^{0}(K)=0$. Also consider the exact local cohomology sequence of the exact sequence (3):

$$
\begin{aligned}
\bigoplus_{i<j} H_{\mathbf{m}}^{i}\left(R\left(-d_{i}-d_{j},-d_{i}^{\prime}-d_{j}^{\prime}\right)\right)_{k, k^{\prime}} & \longrightarrow H_{\mathbf{m}}^{i}(K)_{k, k^{\prime}} \\
& \longrightarrow H_{\mathbf{m}}^{i+1}\left(R\left(-\sum_{i=1}^{3} d_{i},-\sum_{i=1}^{3} d_{i}^{\prime}\right)\right) .
\end{aligned}
$$

$H_{\mathbf{m}}^{1}(K)_{k, k^{\prime}}=0$ if

$$
\begin{array}{r}
\bigoplus_{i<j} H_{\mathbf{m}}^{1}\left(R\left(-d_{i}-d_{j},-d_{i}^{\prime}-d_{j}^{\prime}\right)\right)_{k, k^{\prime}}=0 \text { and } \\
H_{\mathbf{m}}^{2}\left(R\left(-\sum_{i=1}^{3} d_{i},-\sum_{i=1}^{3} d_{i}^{\prime}\right)\right)_{k, k^{\prime}}=0 .
\end{array}
$$

Since we know that $R$ is strongly $(0,0)$-regular (see [8]), (44) holds for all $k, k^{\prime}$, and (5) can be written as the following:

$$
H_{\mathbf{m}}^{2}(R)_{k-\sum_{i=1}^{3} d_{i}, k^{\prime}-\sum_{i=1}^{3} d_{i}^{\prime}}=H^{1}\left(\mathbf{P}^{1} \times \mathbf{P}^{1}, \mathcal{O}_{\mathbf{P}^{1} \times \mathbf{P}^{1}}\left(k-\sum_{i=1}^{3} d_{i}, k^{\prime}-\sum_{i=1}^{3} d_{i}^{\prime}\right)\right)=0 .
$$

Now apply the Künneth formula, [10],

$$
\begin{aligned}
& H^{1}\left(\mathbf{P}^{1} \times \mathbf{P}^{1}, \mathcal{O}_{\mathbf{P}^{1} \times \mathbf{P}^{1}}\left(k-\sum_{i=1}^{3} d_{i}, k^{\prime}-\sum_{i=1}^{3} d_{i}^{\prime}\right)\right) \\
& =\bigoplus_{i+j=1} H^{i}\left(\mathbf{P}^{1}, \mathcal{O}\left(k-\sum_{i=1}^{3} d_{i}\right)\right) \otimes H^{j}\left(\mathbf{P}^{1}, \mathcal{O}\left(k^{\prime}-\sum_{i=1}^{3} d_{i}^{\prime}\right)\right) .
\end{aligned}
$$

By Serre's computation of the cohomology of projective space this last one clearly is 0 , when $k=\sum_{i=1}^{3} d_{i}-1, \forall l$, or $l=\sum_{i=1}^{3} d_{i}^{\prime}-1, \forall k$, or $k>\sum_{i=1}^{3} d_{i}-1$, $l>\sum_{i=1}^{3} d_{i}^{\prime}-1$, or $k>\sum_{i=1}^{3} d_{i}-1, l>\sum_{i=1}^{3} d_{i}^{\prime}-1$, i.e.,

$$
\left(k-\sum_{i=1}^{3} d_{i}+1\right)\left(k^{\prime}-\sum_{i=1}^{3} d_{i}^{\prime}+1\right) \geq 0 .
$$

Therefore, we have

$$
H_{\mathbf{m}}^{1}(K)_{k, k^{\prime}}=0 \text { when }\left(k-\sum_{i=1}^{3} d_{i}+1\right)\left(k^{\prime}-\sum_{i=1}^{3} d_{i}^{\prime}+1\right) \geq 0 .
$$


Since $L \hookrightarrow \bigoplus_{i=1}^{3} R\left(-d_{i},-d_{i}^{\prime}\right), H_{\mathbf{m}}^{0}(L)=0$. This implies that

$$
H_{\mathbf{m}}^{0}(L / K)_{k, k^{\prime}}=0 \text { when }\left(k-\sum_{i=1}^{3} d_{i}+1\right)\left(k^{\prime}-\sum_{i=1}^{3} d_{i}^{\prime}+1\right) \geq 0 \text {. }
$$

Clearly, $\mathbf{m}^{\alpha} L / K=0$ for some $\alpha$, and by Proposition 3.7 and the remark following, this implies that $\operatorname{Supp}(L / K) \subset \mathbb{V}(\mathbf{m})$. It is well known that this last condition implies that $H_{\mathbf{m}}^{0}(L / K)=L / K$, and from (6) this gives $L_{k, k^{\prime}}=K_{k, k^{\prime}}$ when $\left(k-\sum_{i=1}^{3} d_{i}+1\right)\left(k^{\prime}-\sum_{i=1}^{3} d_{i}^{\prime}+1\right) \geq 0$. This means $r=r_{k, k^{\prime}} \in K_{k, k^{\prime}}$ and $K_{k, k^{\prime}}=K_{k, k^{\prime}}^{\mathrm{sat}}$ for those same indices, which was to be proved.

Theorem 3.13. Let $\mathcal{O}_{p}$ be the local ring of a point $p$ in $\mathbf{P}^{1} \times \mathbf{P}^{1}$, and let $\mathcal{I}_{p} \subset \mathcal{O}_{p}$ be a codimension two ideal. Then

$$
\operatorname{dim}_{k} \mathcal{I}_{p} / \mathcal{I}_{p}^{2} \geq 2 \operatorname{dim}_{k} \mathcal{O}_{p} / \mathcal{I}_{p}
$$

Furthermore, equality holds if and only if $\mathcal{I}_{p}$ is a complete intersection in $\mathcal{O}_{p}$.

Proof. See [7, Folgerung 2.6, p. 154].

Theorem 3.14. If $I=\left\langle f_{1}, f_{2}, f_{3}\right\rangle \subset R$ has codimension two, then $K^{\text {sat }}=V$ if and only if $I$ is a local complete intersection.

Proof. Since $K^{\text {sat }}=V \Leftrightarrow H\left(K^{\text {sat }}\right)=H(V)$, we will compute both $H\left(K^{\text {sat }}\right)$ and $H(V)$ :

$$
\begin{gathered}
H(K)=H\left(K^{\mathrm{sat}}\right)=\sum_{i<j} H\left(R\left(-d_{i}-d_{j},-d_{i}^{\prime}-d_{j}^{\prime}\right)\right)-H\left(R\left(-\sum_{i=1}^{3} d_{i},-\sum_{i=1}^{3} d_{i}^{\prime}\right)\right), \\
H\left(K^{\mathrm{sat}}\right)\left(k, k^{\prime}\right)=\sum_{i<j}\left(k-d_{i}-d_{j}+1\right)\left(k^{\prime}-d_{i}^{\prime}-d_{j}^{\prime}+1\right) \\
-\left(k-\sum_{i=1}^{3} d_{i}+1\right)\left(k^{\prime}-\sum_{i-1}^{3} d_{i}^{\prime}+1\right) \\
=\sum_{i=1}^{3}\left(k-d_{i}+1\right)\left(k^{\prime}-d_{i}^{\prime}+1\right)-(k+1)\left(k^{\prime}+1\right) .
\end{gathered}
$$

Now consider $H(V)$. Since $V=S \cap \bigoplus_{i=1}^{3} I^{\text {sat }}\left(-d_{i},-d_{i}^{\prime}\right)$ and

$$
0 \rightarrow S \rightarrow \bigoplus_{i=1}^{3} R\left(-d_{i},-d_{i}^{\prime}\right) \rightarrow I \rightarrow 0
$$

we will have the exact sequence

$$
0 \rightarrow V \rightarrow \bigoplus_{i=1}^{3} I^{\mathrm{sat}}\left(-d_{i},-d_{i}^{\prime}\right) \rightarrow I I^{\mathrm{sat}} \rightarrow 0
$$

Then

$$
H(V)=\sum_{i=1}^{3} H\left(I^{\mathrm{sat}}\left(-d_{i},-d_{i}^{\prime}\right)\right)-H\left(I I^{\mathrm{sat}}\right) .
$$

Since $0 \rightarrow I^{\text {sat }} \rightarrow R \rightarrow R / I^{\text {sat }} \rightarrow 0$ and $V\left(I^{\text {sat }}\right)=V(I)=Z$ is zero dimensional, we have

$$
H\left(I^{\mathrm{sat}}\right)=H(R)-H\left(R / I^{\mathrm{sat}}\right)=H(R)-\operatorname{deg}(Z) .
$$


Therefore

$$
H(V)=\sum_{i=1}^{3} H\left(R\left(-d_{i},-d_{i}^{\prime}\right)\right)-3 \operatorname{deg}(Z)-H\left(I I^{\mathrm{sat}}\right) .
$$

Note that $I^{2}, I I^{\text {sat }}$ have the same saturation. To see this, it is enough to show $I I^{\text {sat }} \subset\left(I^{2}\right)^{\text {sat }}$, since $I^{2} \subset I I^{\text {sat }}$. Let $f \in I I^{\text {sat }}$, so $f=\sum_{i=1}^{k} f_{i} g_{i}$ with $f_{i} \in$ $I, g_{i} \in I^{\text {sat }}$. But there exist an $n$ such that $\langle s t, s v, u t, u v\rangle^{n} g_{i} \subset I$ for all $i$, therefore $f \in\left(I^{2}\right)^{\text {sat }}$. Thus from Remark 3.10, we get $H\left(I^{2}\right)=H\left(I I^{\text {sat }}\right)$. Now

$$
H(V)=\sum_{i=1}^{3} H\left(R\left(-d_{i},-d_{i}^{\prime}\right)\right)-3 \operatorname{deg}(Z)-H\left(I^{2}\right) .
$$

The exact sequence

$$
0 \rightarrow I^{2} \rightarrow R \rightarrow R / I^{2} \rightarrow 0
$$

and

$$
0 \rightarrow I / I^{2} \rightarrow R / I^{2} \rightarrow R / I \rightarrow 0
$$

gives

$$
H\left(I^{2}\right)=H(R)-H\left(R / I^{2}\right)=H(R)-H\left(I / I^{2}\right)-\operatorname{deg}(Z) .
$$

Therefore, we have

$$
\begin{aligned}
H(V)\left(k, k^{\prime}\right)= & \sum_{i=1}^{3} H\left(R\left(-d_{i},-d_{i}^{\prime}\right)\right)\left(k, k^{\prime}\right)-H(R)\left(k, k^{\prime}\right)-2 \operatorname{deg}(Z)+H\left(I / I^{2}\right)\left(k, k^{\prime}\right) \\
= & \sum_{i=1}^{3}\left(k-d_{i}+1\right)\left(k^{\prime}-d_{i}^{\prime}+1\right)-(k+1)\left(k^{\prime}+1\right) \\
& -2 \operatorname{deg}(Z)+H\left(I / I^{2}\right)\left(k, k^{\prime}\right) .
\end{aligned}
$$

Comparing $H\left(K^{\text {sat }}\right)$ and $H(V)$, we see that

$$
H\left(K^{\text {sat }}\right)=H(V) \Longleftrightarrow H\left(I / I^{2}\right)=2 \operatorname{deg}(Z) .
$$

If $\mathcal{I}$ is the ideal sheaf of $Z$, then

$$
\operatorname{deg}(Z)=\operatorname{dim}_{k} H^{0}\left(Z, \mathcal{O}_{Z}\right)=\operatorname{dim}_{k} H^{0}\left(\mathbf{P}^{1} \times \mathbf{P}^{1}, \mathcal{O}_{\mathbf{P}^{1} \times \mathbf{P}^{1}} / \mathcal{I}\right)=\sum_{p \in Z} \operatorname{dim}_{k} \mathcal{O}_{p} / \mathcal{I}_{p}
$$

where $\mathcal{O}_{p}, \mathcal{I}_{p}$ is the localization at $p \in Z$. Since $\mathcal{I} / \mathcal{I}^{2}$ has zero-dimensional support, we have

$$
H\left(I / I^{2}\right)=\operatorname{dim}_{k} H^{0}\left(\mathbf{P}^{1} \times \mathbf{P}^{1}, \mathcal{I} / \mathcal{I}^{2}\right)=\sum_{p \in Z} \operatorname{dim}_{k} \mathcal{I}_{p} / \mathcal{I}_{p}^{2} .
$$

By Theorem 3.13, we know that

$$
\operatorname{dim}_{k} \mathcal{I}_{p} / \mathcal{I}_{p}^{2} \geq 2 \operatorname{dim}_{k} \mathcal{O}_{p} / \mathcal{I}_{p}
$$

for every $p \in Z$ where equality holds if and only if $\mathcal{I}_{p}$ is LCI. Therefore, we have

$$
H\left(I / I^{2}\right)=2 \operatorname{deg}(Z) \Leftrightarrow \operatorname{dim}_{k} \mathcal{I}_{p} / \mathcal{I}_{p}^{2}=2 \operatorname{dim}_{k} \mathcal{O}_{p} / \mathcal{I}_{p}, \quad \forall p \in Z,
$$

and we conclude that

$$
H\left(I / I^{2}\right)=2 \operatorname{deg}(Z) \Leftrightarrow I \text { is LCI. }
$$


Corollary 3.15. If $I=\left\langle f_{1}, f_{2}, f_{3}\right\rangle \subset R$ has codimension two, and the bidegree of $f_{i}$ is $\left(d_{i}, d_{i}^{\prime}\right)$, then

$$
K_{k, k^{\prime}}=V_{k, k^{\prime}} \subset \bigoplus_{i=1}^{3} R\left(-d_{i},-d_{i}^{\prime}\right)_{k, k^{\prime}}
$$

when $\left(k-\sum_{i=1}^{3} d_{i}+1\right)\left(k^{\prime}-\sum_{i=1}^{3} d_{i}^{\prime}+1\right) \geq 0$, if and only if I is a local complete intersection.

Proof. $(\Leftarrow)$ If $I$ is LCI, then $K^{\text {sat }}=V$ by the theorem. But $K_{k, k^{\prime}}=K_{k, k^{\prime}}^{\text {sat }}$ when $\left(k-\sum_{i=1}^{3} d_{i}-1\right)\left(k^{\prime}-\sum_{i=1}^{3} d_{i}^{\prime}+1\right) \geq 0$, by Lemma 3.12

$(\Rightarrow)$ If $K_{k, k^{\prime}}=V_{k, k^{\prime}}$ when $\left(k-\sum_{i=1}^{3} d_{i}-1\right)\left(k^{\prime}-\sum_{i=1}^{3} d_{i}^{\prime}-1\right) \geq 0$, then $H(K)=$ $H\left(K^{\text {sat }}\right)=H(V)$. By Lemma 3.12, we have $K^{\text {sat }}=V$. By Theorem 3.14, we have $I$ is LCI.

Remark 3.16. If $d_{i}=m$ and $d_{i}^{\prime}=n$ for $i=1,2,3$, then Corollary 3.15 implies Proposition 2.3. It is because $K_{3 m-1,3 n-1} \subset \bigoplus_{i=1}^{3} R(-m,-n)$ has pure degree $(2 m-1,2 n-1)$ by Remark 2.2 .

Example 3.17. The following example shows that not all syzygies vanishing at the base point are Koszul syzygies:

$I=\left\langle s^{2} v^{2}, u^{2} t^{2}, s^{2} t^{2}\right\rangle$. The only base point of ideal $I$ is $p=(0: 1 ; 0: 1)$, and $I_{p}=\left\langle s^{2}, t^{2}\right\rangle$. The base point is a local complete intersection. Consider the syzygies $\left(s u t^{4} v, 0,-s u t^{2} v^{3}\right)$ and $\left(0, s^{4} u t v,-s^{2} u^{3} t v\right)$ of bidegree $(2,5)$ and $(5,2)$, respectively. By definition, they vanish on the base point, since $\mathbf{m}\left(s u t^{4} v, 0, s u t^{2} v^{3}\right) \subset I$, $\mathbf{m}\left(0, s^{4} u t v,-s^{2} u^{3} t v\right) \subset I$. But neither one of them is a Koszul syzygy, since the Koszul syzygies are generated by

$$
\left(u^{2} t^{2},-s^{2} v^{2}, 0\right),\left(s^{2} t^{2}, 0,-s^{2} v^{2}\right),\left(0, s^{2} t^{2},-u^{2} t^{2}\right) .
$$

\section{REFERENCES}

[1] D. A. Cox, Equations of parametric curves and surfaces via syzygies, Contemporary Mathematics, 286, (2001), 1-20. MR1874268 (2002i:14056)

[2] D. A. Cox, R. N. Goldman and M. Zhang, On the validity of implicitization by moving quadrics for rational surfaces with no base points, J. Symb. Comput., 29, (2000), 419-440. MR:1751389 (2002d:14098)

[3] D. A. Cox and H. Schenck, Local complete intersections in $\mathbb{P}^{2}$ and Koszul syzygies, Proc. Amer. Math. Soc., 131, (2003), 2007-2014. MR1963743 (2004b:13010)

[4] D. Eisenbud, Commutative Algebra with a View Toward Algebraic Geometry, Graduate Texts in Mathematics, 150, Springer-Verlag, 1995. MR.1322960 (97a:13001)

[5] G.-M. Greuel, G. Pfister and H. Schönemann, Singular 2.0, A Computer Algebra System for Polynomial Computations, Centre for Computer Algebra, University of Kaiserslautern, 2001, http://www.singular.uni-kl.de.

[6] R. Hartshorne, Algebraic Geometry, Graduate Texts in Mathematics, 52, Springer-Verlag, 1977. MR0463157 (57:3116)

[7] J. Herzog, Ein Cohen-Macauly-Kriterium mit Anwedungen auf den Konormalenmodul und den Differentialmodule, Math. Z., 163, (1978), 149-162. MR0512469 (80a:13025)

[8] J. W. Hoffman and H. Wang, Castelnuovo-Mumford regularity in biprojective spaces, Adv. Geom. 4 (2004), 513-536. MR2096526

[9] E. Hyry, The diagonal subring and the Cohen-Macaulay property of a multigraded ring, Trans. Amer. Math. Soc., 351, 6, (1999), 2213-2232. MR1467469 (99i:13005)

[10] J. H. Sampson and G. Washnitzer, A Künneth formula for coherent algebraic sheaves, Illinois J. Math., 3, (1959), 389-402. MR0106906 (21:5636)

[11] J. P. Serre, Local Algebra, Springer-Verlag, 2000. MR1771925 (2001b:13001) 
[12] R. P. Stanley, Combinatorics and commutative algebra, Birkhäuser, 1983. MR0725505 (85b:05002)

[13] H. Wang, Equations of Parametric Surfaces with Base Points via Syzygies, J. Symbolic Comput. 39 (2005), 73-101. MR2168242

Department of Mathematics, Louisiana State University, Baton Rouge, Louisiana 70803

E-mail address: hoffman@math.1su.edu

Department of Mathematics, Southeast Missouri State University, Cape Girardeau, Missouri 63755

E-mail address: hwang@semo.edu 